\title{
Oral Session 6: Sensorimotor Integration
}

\section{6-1 [\#3020]}

\section{Fear of falling: Posture control as influenced by perceived fall height}

\author{
L.R. Young ${ }^{\mathrm{a}}$, L. Bernard-Demanze ${ }^{\mathrm{b}}$, L. Borel ${ }^{\mathrm{b}}$ and M. \\ Lacour $^{\mathrm{b}}$ \\ ${ }^{a}$ Man Vehicle Laboratory, MIT, Cambridge, MA 02139, \\ USA \\ ${ }^{\mathrm{b}}$ CNRS-UMR 6146 CNRS/Université de Provence, \\ Marseille, France
}

Human control of posture, developed to permit standing and locomotion in Earth's gravity, can be adapted to meet a variety of environmental challenges. Whether posture is associated with an EVA looking at the Earth from orbit, locomotion on the moon, or simply standing on a moving surface on the ground, the strategy for maintaining balance is influenced by the perception of the consequence of falling. We examined the posture mechanisms for three groups of subjects who attempted to maintain balance on a moving platform, first at ground level and then elevated. The subjects were young normals, elderly normals, or patients recovering from hemi-labyrinthectomy. We observed an alteration of strategy, especially among the elderly and the patients, when we either increased the task difficulty, by eye closure, or when we increased the consequence of falling by raising the platform. Subjects with a fear of falling reverted to a "rigidification" strategy which caused the head to be stabilized over the moving platform, as opposed to an inertial compensation strategy in which the feet and hips oscillated under an inertially fixed head.

Acknowledgement: Supported by MIT (MISTI; MIT-France) and grants from the Ministère de Enseignement Supérieur et de la Recherche (CNRS UMR 6149).
6-2 [\#3021]

\author{
Effects of mechanostimulation of the foot sole \\ cutaneous receptors on characteristics of leg \\ muscles stretch reflexes under conditions of "Dry \\ Immersion"
}

I.B. Kozlovskaya, I.B. Saenko, A.Z. Zakirova and T.A. Shigueva

RF SCC Institute for Biomedical Problems RAS, 76A Khoroshevskoe shosse, Moscow, 123007, Russia

Effects of the mechanostimulation of deep cutaneous receptors of the sole on the recruitment and the recovery curves of m.soleus (S) and m.gastrocnemius lateralis (GL) stretch reflexes have been studied under conditions of dry immersion (DI).

18 volunteers $22-30$ years of age that signed the informational content to participate in the study have been exposed to 7-day DI, being divided in 2 groups control (C) and experimental (E) ones. Subjects of $\mathrm{E}$ group when being in immersion received every day the mechanical stimulation of the support zones of the soles, that mimicks all the parameters of slow and fast pacing. The overall duration of the stimulation, produced by Compensator of Support Unloading (KOR), was 2 hours per day. The recruitment and the recovery curves of muscles under study have been recorded pre-, during (on the days 3 and 7) and postimmersion (also on the days 3 and 7 ). To obtain the recovery curve the paired stimulation procedure has been used with interval durations between conditioning and testing stimuli from 10 to $3000 \mathrm{~ms}$ and with the reflex amplitudes of both equal to $40-60 \%$ of the maximal one.

The results of studies of the H-reflexes recovery curves revealed a pronounced facilitation effect of the cutaneous foot sole afferentation that was manifested by a significant decline of the curves amplitude under conditions of support unloading in the $\mathrm{C}$ group and its enhancement in E group in which the mechanostimulation of the foot sole support zones during DI has been 
used. The changes of characteristics of the recruitment curves, on the opposite, pointed out to inhibitory effects of the foot sole stimulation on the excitability of the leg muscles stretch reflexes: the absolute and relative values of reflexes maximal amplitudes in this case have increased in DI in "no stimulation" control group and didn't change in the E group.
Thus, the results of studies have shown that cutaneous afferents of the soles interact strongly with spinal interneuronal circuits and that the effects of this interaction could be both excitatory or inhibitory depending on testing protocol. 